\title{
LASER LIGHT EFFECT ON DORSAL ROOT GANGLIA NEURONS: A PRE-CLINICAL STUDY
}

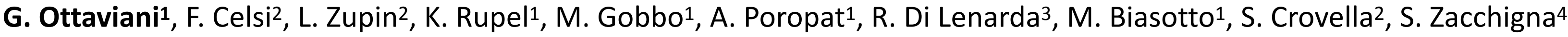 \\ 1 University of Trieste, Dental Science - Oral Medicine and Pathology, Trieste, Italy \\ 2 I.R.C.C.S. materno infantile Burlo Garofolo, Immunology Lab., Trieste, Italy \\ 3 University of Trieste, Department of Medical- Surgical and Health Sciences, Trieste, Italy \\ 4 International Centre for Genetic Engineering and Biotechnology ICGEB, Cardiovascular Biology, Trieste, Italy
}

\section{Introduction}

In oncological patients, affected by oral mucositis (OM), pain worsens the quality of life, often requiring high doses of analgesic drugs, and could lead to the halting of the therapies, with significant negative impact on their cancer prognosis. Supportive care and pain management due to OM has significant economic impacts: LT represents an excellent resource as supportive therapy with direct implication for improving cancer patient care.

\section{Methods}

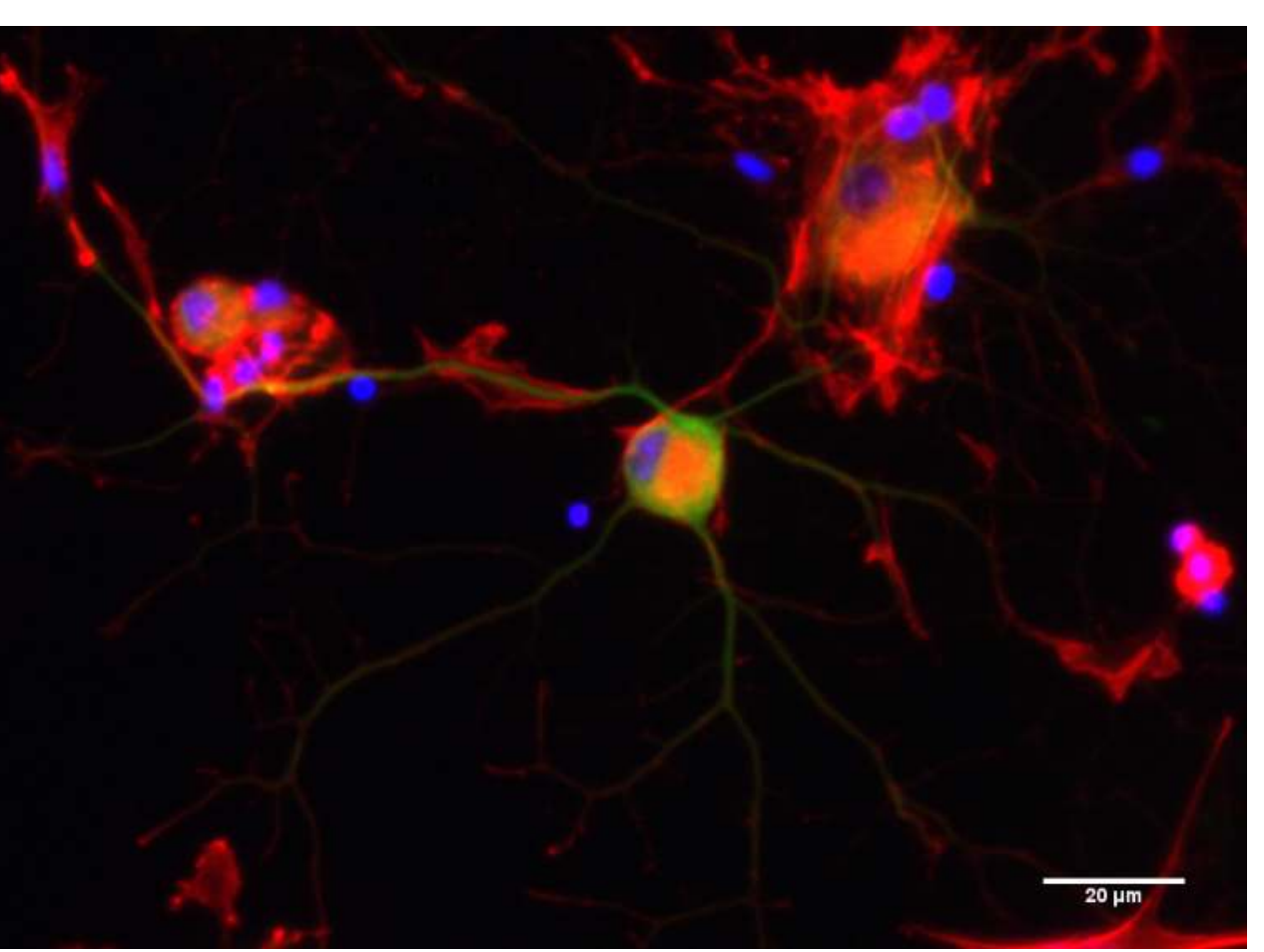

Figure 1: Representative fluorescent immunocytochemistry for DRG cultures.
Primary culture of murine sensory neurons, derived from dorsal root ganglia (DRG; Figure 1), were employed in the experiments. The following markers have been employed to stain neurons: F-actin (red), general cellular marker; beta-III tubulin (green), neuronal cells marker, and DAPI (blue) to stain nuclei. The cells were irradiated with two laser protocols: $\lambda 800$ and $970 \mathrm{~nm}, 0.1 \mathrm{~W} / \mathrm{cm} 2,6 \mathrm{~J} / \mathrm{cm} 2,5 \mathrm{~Hz}$. Immediately after irradiation, cells were analysed for production of ATP, radical oxygen species (ROS) and superoxide anion.

DIODE LASER TREATMENTS
L1: $\lambda$ 800, $0.1 \mathrm{~W} / \mathrm{cm} 2,6 \mathrm{~J} / \mathrm{cm} 2,5 \mathrm{~Hz}$.
$\mathrm{L} 2: \lambda 970,0.1 \mathrm{~W} / \mathrm{cm} 2,6 \mathrm{~J} / \mathrm{cm} 2,5 \mathrm{~Hz}$.
Class IV, K-Laser Cube series, Eltech
K-Laser, Via Castagnole $20 / \mathrm{H}$,
Treviso, (Italy)

\section{Objectives}

This study aimed at exploring the analgesic activities of LT in an in vitro model of sensory neurons, analyzing cellular parameters possibly correlated to nociceptive pathways.

\section{Results}

Both the laser protocols were able to decrease the production of ATP and to increment ROS concentration, meanwhile only the laser therapy set at $800 \mathrm{~nm}$ wavelength increased the level of superoxide anion (Figure 2).

A

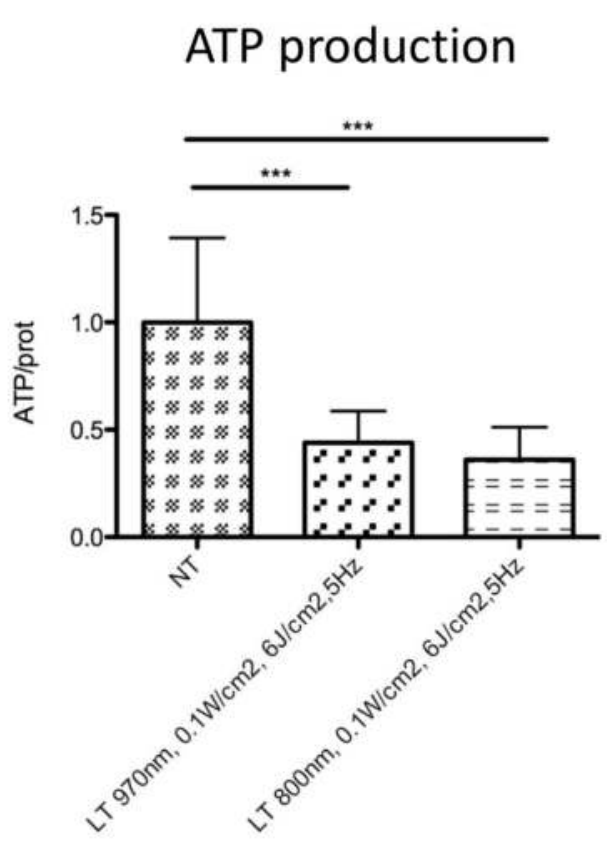

B

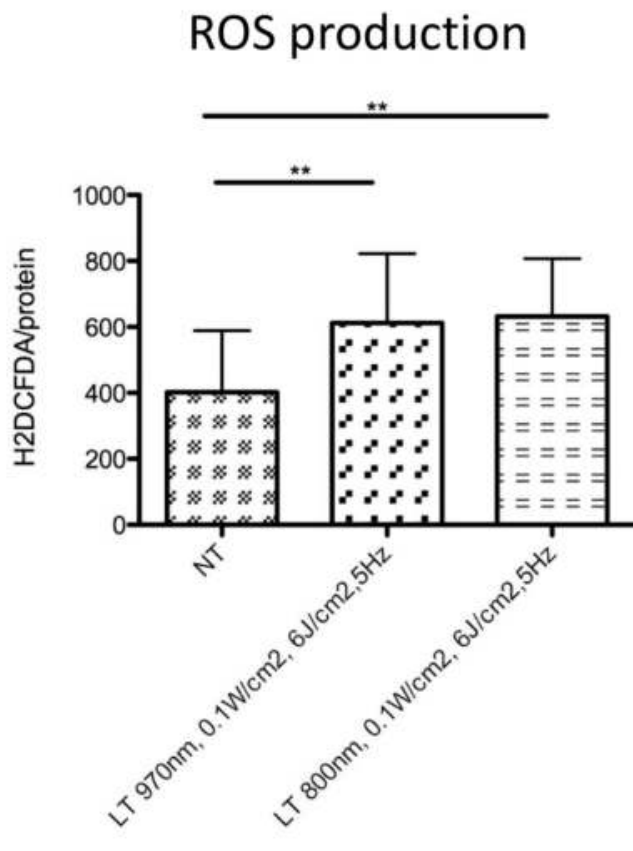

C Superoxide anion production

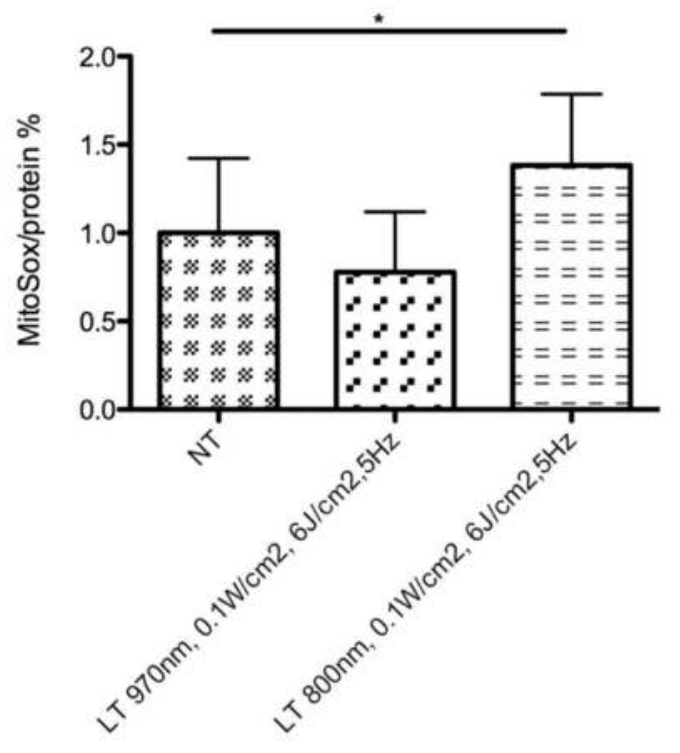

Figure 2. A. ATP production in DRG cultures exposed to laser therapy, expressed as micromole ATP vs total protein content. B. ROS generation after laser therapy measured by Arbitrary Fluorescent Unit (A.F.U.) of 2', 7'-Dichlorofluorescin diacetate (H2DCFDA) vs total protein content. C. Superoxide anion production after laser therapy as measured by A.F.U. of MitoSox vs total protein content (in percentage). Results are expressed as mean \pm S.E.M., ANOVA followed by Bonferroni correction was conducted to determine statistical significance of the data and horizontal bars indicate statistical significant differences vs NT DRG cultures, with ${ }^{*} p<0.05$ and ${ }^{* *} p<0.01$.

\section{Conclusion}

Our results highlight a direct effect of laser therapy on DRG neurons, specifically it appears to inhibit cellular metabolism, lowering ATP production and promoting ROS generation, thus suggesting a stress response to the nociceptive stimulus.

Finally, the differential outcome on superoxide anion could be due to the specific target of $\lambda 800 \mathrm{~nm}$ on cytochrome $C$ oxidase enzyme. These findings suggest a direct and immediate metabolic effect of laser light on DRG neurons. 